\title{
OPEN Changes in vitamin D levels and depressive symptoms in later life in England
}

\author{
Giorgio Di Gessa ${ }^{\bowtie}$, Jane P. Biddulph, Paola Zaninotto \& Cesar de Oliveira
}

Inadequate vitamin D levels have been associated with increased risk of depression. However, most of these studies are cross-sectional and failed to investigate the effect of changes in vitamin D levels. This study aimed to investigate the longitudinal association of changes in serum 25 -hydroxyvitamin D levels with depressive symptoms in 3365 participants of the English Longitudinal Study of Ageing, a large nationally-representative study of older adults. Based on their vitamin D levels at baseline and follow-up (sufficient $\geq 50 \mathrm{nmol} / \mathrm{L}$; insufficient $<50 \mathrm{nmol} / \mathrm{L}$ ), participants were classified as follows: with sufficient levels at both waves; with sufficient levels at baseline but not at follow-up; with insufficient levels at baseline but $\geq 50 \mathrm{nmol} / \mathrm{L}$ at follow-up; and with levels $<50 \mathrm{nmol} / \mathrm{L}$ at each time point. Depressive symptoms were measured using the 8-point CES-D scale. Data were analysed using logistic regression models. Compared with those with sufficient levels of vitamin D at both waves, only those with insufficient levels throughout were more likely to report elevated depressive symptoms $(\mathrm{OR}=1.39,95 \% \mathrm{Cl}=1.00-1.93)$. Becoming or no longer being vitamin $\mathrm{D}$ deficient was, in the short term, not associated with elevated depressive symptoms. Further evidence is required on whether vitamin D supplementation might contribute to the prevention or treatment of depression as well as on the duration of time for changes in vitamin $D$ levels to lead to detectable changes in depressive symptoms.

It is estimated that depression affects more than 300 million people worldwide and that it is the world's leading cause of disability ${ }^{1}$. Depression is also associated with higher mortality, reduced productivity, and increased direct and indirect healthcare costs ${ }^{2-4}$. Although depression aetiology and pathophysiology have not yet been fully explained, recent studies highlight the potential role of new biological factors that may affect mood. For instance, the presence of vitamin D receptors in the brain and its key role in the responses of the immune and nervous systems make it biologically plausible for vitamin $\mathrm{D}$ to be associated with brain activity and thus depression $^{5}$, with hypovitaminosis D i.e. insufficiency or deficiency of vitamin D indicating an underlying biological susceptibility for depression ${ }^{6}$.

Hypovitaminosis D is a main public health problem worldwide. It is estimated that about 1 billion people worldwide have vitamin $\mathrm{D}$ deficiency, while $50 \%$ of the population has vitamin $\mathrm{D}$ insufficiency ${ }^{7}$ with a high prevalence of hypovitaminosis D also found in sunny climates ${ }^{8,9}$. Deficiency of 25-hydroxyvitamin D [25(OH)D], the major circulating form of vitamin $\mathrm{D}$, is a widespread disorder across all age groups ${ }^{10}$. Older adults, however, are particularly at risk of hypovitaminosis $\mathrm{D}$ given that vitamin $\mathrm{D}$ absorption from food as well as skin synthesis by UVB-irradiation from sun exposure become less efficient with increasing age $e^{9,11}$.

Although vitamin D is well-known to be essential to maintain serum calcium and phosphorus levels in a healthy physiologic range for musculoskeletal health, observational studies have also found low vitamin D to be associated with a range of non-skeletal health conditions $s^{9,12-14}$ including symptoms of depression. Early work by Hoogendijk and colleagues ${ }^{15}$ using data from the Longitudinal Aging Study Amsterdam found that, among the 1282 adults aged 65-95 years, depressed subjects showed 25(OH)D levels that were 14\% lower than non-depressed individuals (assessed using the Centre for Epidemiologic Studies Depression scale). With a few exceptions $s^{16,17}$, similar results have been found in other cross-sectional studies that have investigated the associations between depressive symptoms and vitamin D levels across different age groups, settings, and both among healthy non-institutionalised populations and in patients with medical comorbidities ${ }^{18-24}$.

However, cross-sectional studies do not allow determination of whether hypovitaminosis D precedes the onset of depression or whether individuals have low 25(OH)D levels because they are depressed. Cohort studies have tried to shed light on this issue exploring longitudinally the relationship between vitamin $\mathrm{D}$ and depression. A 


\begin{tabular}{|c|c|c|c|c|c|c|}
\hline & \multicolumn{4}{|c|}{ All seasons } & \multicolumn{2}{|l|}{ Baseline season } \\
\hline & $\%(n)$ & $\begin{array}{l}\text { Mean vitamin D level at } \\
\text { baseline } \\
\text { nmol/L (SD) }\end{array}$ & $\begin{array}{l}\text { Mean vitamin D level at } \\
\text { follow-up } \\
\text { nmol/L(SD) }\end{array}$ & $\begin{array}{l}\text { Mean difference in vitamin } \\
\text { D level } \\
\text { nmol/L(SD) }\end{array}$ & $\begin{array}{l}\text { Summer and Autumn } \\
\%(\mathbf{n})\end{array}$ & $\begin{array}{l}\text { Winter and Spring } \\
\%(\mathbf{n})\end{array}$ \\
\hline \multicolumn{7}{|c|}{ Vitamin D transition category } \\
\hline $\begin{array}{l}25(\mathrm{OH}) \mathrm{D}>50 \mathrm{nmol} / \mathrm{L} \text { at } \\
\text { baseline and follow-up }\end{array}$ & $\begin{array}{l}31.1 \\
(1045)\end{array}$ & $\begin{array}{l}73.1 \\
(18.2)\end{array}$ & $\begin{array}{l}71.6 \\
(16.5)\end{array}$ & \begin{tabular}{|l|}
-1.5 \\
$(19.5)$ \\
\end{tabular} & \begin{tabular}{|l|}
35.1 \\
$(787)$
\end{tabular} & \begin{tabular}{|l|}
23.2 \\
$(258)$
\end{tabular} \\
\hline $\begin{array}{l}25(\mathrm{OH}) \mathrm{D} \leq 50 \mathrm{nmol} / \mathrm{L} \text { at } \\
\text { baseline and follow-up }\end{array}$ & $\begin{array}{l}37.4 \\
(1255)\end{array}$ & $\begin{array}{l}32.2 \\
(10.6)\end{array}$ & $\begin{array}{l}30.8 \\
(10.9)\end{array}$ & \begin{tabular}{|l|}
-1.3 \\
$(11.9)$
\end{tabular} & \begin{tabular}{|l|}
33.0 \\
$(739)$
\end{tabular} & \begin{tabular}{|l|}
46.3 \\
$(516)$
\end{tabular} \\
\hline $\begin{array}{l}25(\mathrm{OH}) \mathrm{D}>50 \text { only at } \\
\text { baseline }\end{array}$ & $\begin{array}{l}15.1 \\
(506)\end{array}$ & $\begin{array}{l}63.4 \\
(12.5)\end{array}$ & $\begin{array}{l}37.0 \\
(9.6)\end{array}$ & $\begin{array}{l}-26.4 \\
(15.4)\end{array}$ & $\begin{array}{l}18.4 \\
(413)\end{array}$ & $\begin{array}{l}8.3 \\
(93)\end{array}$ \\
\hline $\begin{array}{l}25(\mathrm{OH}) \mathrm{D}>50 \text { only at } \\
\text { follow-up }\end{array}$ & $\begin{array}{l}16.4 \\
(549)\end{array}$ & $\begin{array}{l}35.6 \\
(10.0)\end{array}$ & $\begin{array}{l}66.1 \\
(14.2)\end{array}$ & $\begin{array}{l}+30.5 \\
(18.0)\end{array}$ & \begin{tabular}{|l}
13.5 \\
$(301)$
\end{tabular} & $\begin{array}{l}22.2 \\
(248)\end{array}$ \\
\hline All & $\begin{array}{l}100 \\
(3355)\end{array}$ & $\begin{array}{l}50.20^{1} \\
(23.0)\end{array}$ & $\begin{array}{l}50.23^{2} \\
(22.9)\end{array}$ & $\begin{array}{l}0.03 \\
(22.8)\end{array}$ & $\begin{array}{l}100 \\
(2240)\end{array}$ & $\begin{array}{l}100 \\
(1115)\end{array}$ \\
\hline
\end{tabular}

Table 1. Changes in hypovitaminosis D and mean vitamin D level at baseline and follow-up-all sample and by baseline season. ${ }^{1}$ Mean vitamin D level in Summer and Autumn samples at baseline was $54.3 \mathrm{nmol} / \mathrm{L}$; in Winter and Spring samples at baseline was $41.9 \mathrm{nmol} / \mathrm{L} .{ }^{2}$ Mean vitamin D level in Summer and Autumn samples at follow-up was $54.9 \mathrm{nmol} / \mathrm{L}$; in Winter and Spring samples at follow-up was $45.3 \mathrm{nmol} / \mathrm{L}$. Source: ELSA waves 6,8 , and 9 . Analyses restricted to the sample with no missing values in any of the covariates used in the regression models.

2013 meta-analysis of three cohort studies reported mixed findings, depending on the categories and cut-off points being compared ${ }^{25}$. Other recent population-based longitudinal studies on both older men and women as well as adolescents also yielded inconclusive results ${ }^{26-28}$. Using data from the UK Biobank collected on participants aged between 40 and 69 years, Ronaldson and colleagues ${ }^{29}$ also found that vitamin D insufficiency at baseline was associated with depression at follow-up. All these longitudinal studies, however, have only examined the associations between vitamin D levels measured at one point in time (generally at baseline) and either changes in depression or depression at follow-up, failing to account for changes in vitamin D levels over time. This is an important oversight as seasonal changes in vitamin $\mathrm{D}$ levels are quite common ${ }^{30}$. Understanding whether changes in vitamin D levels are associated with changes in depressive symptoms helps to establish whether vitamin D plays a role in the prevention and/or treatment of depression. Thus, using data from the English Longitudinal Study of Ageing (ELSA), we aimed to examine whether and how changes in Vitamin D are associated with depressive symptoms among older men and women.

\section{Results}

Overall levels of Vitamin D and changes over time. Table 1 provides an overview of Vitamin D levels at baseline and follow-up, including changes over time and details by seasons. Overall, the average level of $25(\mathrm{OH}) \mathrm{D}$ was similar at both baseline and follow-up (at $50.2 \mathrm{nmol} / \mathrm{L}$ ). As expected, both at baseline and follow-up, mean serum 25(OH)D concentration levels were insufficient when blood samples were collected in winter and spring compared to summer and autumn. When we looked at changes over time, $37 \%$ of ELSA respondents had insufficient levels of Serum $25(\mathrm{OH}) \mathrm{D}$ at both baseline and follow-up, with $31 \%$ presenting values $>50 \mathrm{nmol} / \mathrm{L}$ at both waves. The percentage of respondents with sufficient levels at baseline but not at follow-up (15\%) was similar to that of respondents whose 25(OH)D increased over $50 \mathrm{nmol} / \mathrm{L}$ over time (16\%). A seasonality effect can be seen with regards to both baseline values and changes: $46 \%$ of the sample whose blood was collected in the summer or autumn at baseline had insufficient levels of vitamin D at baseline compared to $69 \%$ of respondents whose initial interviews were in winter or spring.

Baseline characteristics by follow-up depressive symptoms. Table 2 shows baseline characteristics by depressive symptoms at follow-up. Ten percent of the sample reported four or more depressive symptoms at follow-up, with almost half of respondents depressed at follow up having serum 25(OH)D levels below $50 \mathrm{nmol} / \mathrm{L}$ at both waves. Women, unpartnered respondents, those with lower levels of wealth, sedentary lifestyle, smokers, participants who reported more chronic diseases or ADL or IALD disabilities, those with high waist circumferences as well as participants with elevated depressive symptoms at baseline were more likely to report elevated depressive symptoms at follow-up (see Table 2 for full details).

Multivariate analyses. Odds ratios for the associations between changes in vitamin D levels and elevated depressive symptoms at follow-up are presented in Table 3. In the unadjusted analyses (Model 1) low levels of $25(\mathrm{OH}) \mathrm{D}$ at both baseline and follow-up were significantly associated with elevated depressive symptoms $(\mathrm{OR}=1.97,95 \% \mathrm{CI}=1.48-2.64)$. Moreover, those whose levels of vitamin $\mathrm{D}$ were insufficient only at baseline had higher odds of reporting elevated depressive symptoms at follow-up $(\mathrm{OR}=1.6095 \% \mathrm{CI}=1.12-2.30)$ compared with those with $25(\mathrm{OH}) \mathrm{D}$ levels above $50 \mathrm{nmol} / \mathrm{L}$ at both waves. After controlling for socio-demographic, economic, and health characteristics, only participants with hypovitaminosis D at both waves remained significantly more likely to have elevated depressive symptoms at follow-up than those with sufficient levels even, although the $\mathrm{OR}$ attenuated from Models $2(\mathrm{OR}=1.96)$ to Model $5(\mathrm{OR}=1.49)$. Even after controlling for ele- 


\begin{tabular}{|c|c|c|c|}
\hline & CES-D score $<4$ & $\geq 4$ CES-D symptoms & $P$ value \\
\hline \multicolumn{4}{|l|}{ Change in vitamin D levels (\%) } \\
\hline $25(\mathrm{OH}) \mathrm{D}>50 \mathrm{nmol} / \mathrm{L}$ at both waves & 32.1 & 21.9 & \multirow{4}{*}{$<0.001$} \\
\hline $25(\mathrm{OH}) \mathrm{D} \leq 50 \mathrm{nmol} / \mathrm{L}$ at both waves & 36.2 & 48.7 & \\
\hline $25(\mathrm{OH}) \mathrm{D}>50$ only at baseline & 15.5 & 11.7 & \\
\hline $25(\mathrm{OH}) \mathrm{D}>50$ only at follow-up & 16.2 & 17.7 & \\
\hline Mean age (SD) & $65.4(8.31)$ & $65.6(8.96)$ & 0.661 \\
\hline$\%$ Men & 46.1 & 29.4 & $<0.001$ \\
\hline Wave 8 follow-up interview & 66.7 & 68.5 & 0.495 \\
\hline Partnered & 70.7 & 53.5 & $<0.001$ \\
\hline \multicolumn{4}{|l|}{ Education } \\
\hline Low & 33.4 & 43.2 & \multirow{3}{*}{$<0.001$} \\
\hline Medium & 46.3 & 44.5 & \\
\hline High & 20.3 & 12.3 & \\
\hline \multicolumn{4}{|l|}{ Wealth quintiles } \\
\hline Lowest & 12.5 & 27.0 & \multirow{5}{*}{$<0.001$} \\
\hline 2nd & 14.8 & 24.0 & \\
\hline $3 \mathrm{rd}$ & 21.1 & 21.0 & \\
\hline 4th & 24.7 & 16.9 & \\
\hline Highest & 26.9 & 11.1 & \\
\hline \multicolumn{4}{|l|}{ Physical activity } \\
\hline Sedentary or low & 7.5 & 22.5 & \multirow{3}{*}{$<0.001$} \\
\hline Moderate & 42.7 & 50.5 & \\
\hline Vigorous & 49.8 & 27.0 & \\
\hline \multicolumn{4}{|l|}{ Smoking status } \\
\hline Never smoked & 40.1 & 33.3 & \multirow{3}{*}{$<0.001$} \\
\hline Former smoker & 51.1 & 49.3 & \\
\hline Current smoker & 8.7 & 17.4 & \\
\hline Mean number of comorbidities (SD) & $1.02(1.04)$ & $1.61(1.29)$ & $<0.001$ \\
\hline Mean number of ADLs or IADLs (SD) & $0.31(1.00)$ & $1.26(1.99)$ & $<0.001$ \\
\hline \multicolumn{4}{|l|}{ Waist } \\
\hline Low & 23.7 & 18.0 & \multirow{3}{*}{$<0.001$} \\
\hline Medium & 26.5 & 20.1 & \\
\hline High & 49.8 & 61.9 & \\
\hline$\geq 4$ CES-D symptoms at baseline & 6.5 & 41.4 & $<0.001$ \\
\hline Mean number of baseline depressive symptoms (SD) & $0.89(1.43)$ & $3.27(2.56)$ & $<0.001$ \\
\hline Mean number of depressive symptoms at follow-up (SD) & $0.75(0.94)$ & $5.26(1.31)$ & $<0.001$ \\
\hline History of doctor-diagnosed depression & 5.7 & 19.5 & $<0.001$ \\
\hline Summer or Autumn & 33.5 & 30.6 & 0.284 \\
\hline Total \% (and N) of respondents & $90.1(3022)$ & $9.9(333)$ & \\
\hline
\end{tabular}

Table 2. Baseline characteristics by depressive symptoms at follow-up. Source: ELSA waves 6, 8, and 9. Analyses restricted to the sample with no missing values in any of the covariates used in the regression models.

vated depressive symptoms at baseline and seasonality of interviews (Model 7), those with insufficient levels of vitamin D were more likely to report elevated depressive symptoms at follow-up compared with those with $25(\mathrm{OH}) \mathrm{D}$ levels above $50 \mathrm{nmol} / \mathrm{L}$ at both waves $(\mathrm{OR}=1.39,95 \% \mathrm{CI}=1.00-1.93)$. Across all models, those who became at risk (i.e. whose $25(\mathrm{OH}) \mathrm{D}$ levels dropped below $50 \mathrm{nmol} / \mathrm{L}$ ) were not associated with elevated depressive symptoms at follow-up.

Sensitivity analyses (Table 4) were used to further check the robustness of our results. Results showed that even taking into account changes in partnership, employment, wealth, and health (Model A), we found that respondents with low vitamin D levels across waves had significantly higher odds of elevated depressive symptoms at follow-up $(\mathrm{OR}=1.46,95 \% \mathrm{CI}=1.05-2.03)$. Similarly, when we restricted the sample to participants who were interviewed in the same season at both baseline and follow-up (Model B), those with hypovitaminosis D across waves were significantly more likely to report elevated depressive symptoms $(\mathrm{OR}=1.52,95 \% \mathrm{CI} 1.01-2.29)$ than those with $25(\mathrm{OH}) \mathrm{D}$ levels above $50 \mathrm{nmol} / \mathrm{L}$ at both interviews. When using the number of timepoints $(0$, 1 , or 2) 'at risk' through insufficient levels of Vitamin D, we found that having $25(\mathrm{OH}) \mathrm{D}$ levels $\leq 50 \mathrm{nmol} / \mathrm{L}$ only once (that is either at baseline or follow-up) was not associated with elevated depressive symptoms at follow-up. Furthermore, when baseline levels of vitamin $\mathrm{D}$ and absolute changes were considered, we found that absolute changes in $25(\mathrm{OH}) \mathrm{D}$ were not associated with elevated depressive symptoms at follow-up. Results suggesting 


\begin{tabular}{|c|c|c|c|c|c|c|c|}
\hline & \begin{tabular}{|l} 
Model 1 \\
(unadjusted)
\end{tabular} & Model 2 & Model 3 & Model 4 & Model 5 & Model 6 & Model 7 \\
\hline & OR $(95 \% \mathrm{CI})$ & OR (95\% CI) & OR $(95 \% \mathrm{CI})$ & OR (95\% CI) & OR (95\% CI) & OR (95\% CI) & OR $(95 \% \mathrm{CI})$ \\
\hline \multicolumn{8}{|c|}{ Changes in $25(\mathrm{OH}) \mathrm{D}$} \\
\hline$>50$ at both & Reference & Reference & Reference & Reference & Reference & Reference & Reference \\
\hline$\leq 50$ at both & $1.97(1.48-2.64)$ & $\begin{array}{l}1.96(1.47- \\
2.62)\end{array}$ & $\begin{array}{l}1.63(1.21- \\
2.20)\end{array}$ & $\begin{array}{l}1.48(1.09- \\
2.02)\end{array}$ & $\begin{array}{l}1.49(1.09- \\
2.04)\end{array}$ & $\begin{array}{l}1.36(0.98- \\
1.88)\end{array}$ & $1.39(1.00-1.93)$ \\
\hline Became at risk & $1.11(0.74-1.67)$ & $\begin{array}{l}1.11(0.74- \\
1.67)\end{array}$ & $\begin{array}{l}1.01(0.67- \\
1.53)\end{array}$ & $\begin{array}{l}1.03(0.68- \\
1.58)\end{array}$ & $\begin{array}{l}1.04(0.68- \\
1.58)\end{array}$ & $\begin{array}{l}1.05(0.68- \\
1.62)\end{array}$ & $1.05(0.68-1.62)$ \\
\hline $\begin{array}{l}\text { No longer at } \\
\text { risk }\end{array}$ & $1.60(1.12-2.30)$ & $\begin{array}{l}1.56(1.09- \\
2.24)\end{array}$ & $\begin{array}{l}1.35(0.93- \\
1.96)\end{array}$ & $\begin{array}{l}1.30(0.89- \\
1.90)\end{array}$ & $\begin{array}{l}1.30(0.89- \\
1.91)\end{array}$ & $\begin{array}{l}1.17(0.79- \\
1.74)\end{array}$ & $1.17(0.79-1.74)$ \\
\hline
\end{tabular}

Table 3. Unadjusted and fully adjusted ORs (with $95 \%$ CIs) for logistic regression analyses investigating the association between changes in vitamin $\mathrm{D}$ levels and elevated depressive symptoms at follow-up $(\mathrm{N}=3355)$. $\mathrm{OR}=$ Odds Ratio; $\mathrm{CI}=$ Confidence Interval. Nested models, with Model 1 unadjusted; Model $2=$ Model $1+$ adjusted for age, sex and the wave at follow-up; Model $3=$ Model $2+$ adjusted for partnership, paid work, education, and wealth. Model $4=$ Model $3+$ adjusted for health behaviours, health conditions, and functional limitations; Model $5=$ Model $4+$ adjusted for waist circumference; Model $6=$ Model $5+$ baseline elevated depressive symptoms and history of doctor-diagnosed depression; Model $7=$ Model $6+$ season of blood collection at follow-up and an indicator of same seasonality across waves. Source: ELSA waves 6, 8, and 9.

\begin{tabular}{|c|c|c|c|c|c|c|}
\hline & \multicolumn{2}{|l|}{ Model A } & \multicolumn{2}{|l|}{ Model B } & \multicolumn{2}{|l|}{ Model C } \\
\hline & Unadjusted & Fully adjusted & Unadjusted & Fully adjusted & Unadjusted & Fully adjusted \\
\hline & OR $(95 \% \mathrm{CI})$ & OR $(95 \% \mathrm{CI})$ & OR $(95 \% \mathrm{CI})$ & OR $(95 \% \mathrm{CI})$ & OR $(95 \% \mathrm{CI})$ & OR $(95 \% \mathrm{CI})$ \\
\hline \multicolumn{7}{|l|}{ Changes in $25(\mathrm{OH}) \mathrm{D}$} \\
\hline$>50 \mathrm{nmol} / \mathrm{L}$ at both & Reference & Reference & Reference & Reference & Reference & Reference \\
\hline$\leq 50 \mathrm{nmol} / \mathrm{L}$ at both & $1.97(1.48-2.64)$ & $1.46(1.05-2.03)$ & $2.20(1.54-3.14)$ & $1.52(1.01-2.29)$ & $2.21(1.48-3.27)$ & $1.93(1.27-2.94)$ \\
\hline Became at risk & $1.11(0.74-1.67)$ & $1.05(0.68-1.63)$ & $1.40(0.85-2.32)$ & $1.29(0.75-2.23)$ & $1.19(0.69-2.06)$ & $1.14(0.64-2.00)$ \\
\hline No longer at risk & $1.60(1.12-2.30)$ & $1.27(0.84-1.89)$ & $1.67(1.07-2.62)$ & $1.27(0.77-2.11)$ & $1.70(1.03-2.78)$ & $1.56(0.93-2.59)$ \\
\hline \multicolumn{7}{|c|}{$\mathrm{N}$ of times at risk of hypovitaminosis $\mathrm{D}$} \\
\hline Never & Reference & Reference & Reference & Reference & Reference & Reference \\
\hline Once & $1.36(0.99-1.87)$ & $1.17(0.82-1.66)$ & $1.55(1.05-2.30)$ & $1.27(0.83-1.95)$ & $1.44(0.94-2.23)$ & $1.35(0.86-2.11)$ \\
\hline Twice & $1.97(1.48-2.64)$ & $1.46(1.05-2.03)$ & $2.20(1.54-3.14)$ & $1.52(1.01-2.29)$ & $2.21(1.48-3.27)$ & $1.93(1.27-2.94)$ \\
\hline \multicolumn{7}{|c|}{ Baseline 25(OH)D and absolute change } \\
\hline$>50 \mathrm{nmol} / \mathrm{L}$ & Reference & Reference & Reference & Reference & Reference & Reference \\
\hline$\leq 50 \mathrm{nmol} / \mathrm{L}$ & $1.77(1.39-2.34)$ & $1.35(1.01-1.81)$ & $1.77(1.36-2.29)$ & $1.37(0.95-1.97)$ & $2.01(1.41-2.85)$ & $1.79(1.23-2.59)$ \\
\hline $\begin{array}{l}\text { Difference in } 25(\mathrm{OH}) \mathrm{D} \\
\text { over time }\end{array}$ & $1.00(0.99-1.01)$ & $1.00(0.99-1.01)$ & $1.00(0.99-1.01)$ & $1.00(0.99-1.01)$ & $1.00(0.99-1.01)$ & $1.00(0.99-1.01)$ \\
\hline $\mathrm{N}$ respondents & \multicolumn{2}{|l|}{3324} & \multicolumn{2}{|l|}{2251} & \multicolumn{2}{|l|}{2863} \\
\hline
\end{tabular}

Table 4. Unadjusted and fully adjusted ORs (with 95\% CIs) for the relationship between three different indicators of changes in vitamin D levels and elevated depressive symptoms at follow-up under different model assumptions. OR odds ratio, CI confidence interval. Model A refers to analyses which adjust for changes in respondents' partnership, employment, wealth, and health instead of considering these variables only at baseline. The fully-adjusted Model A also adjusts for age, sex, wave at follow-up, education, waist circumference, baseline elevated depressive symptoms, history of doctor-diagnosed depression, season of blood collection at follow-up, and an indicator of same seasonality across waves. Model B only considers those whose blood samples were collected in the same season (winter or spring vs summer or autumn) at both baseline and follow-up. The fully adjusted Model B adjusts for age, sex, wave at follow-up, partnership, paid work, education, wealth, health behaviours, health conditions, functional limitations, waist circumference, baseline elevated depressive symptoms, history of doctor-diagnosed depression and season of blood collection at follow-up. Model C only considers those with no elevated depressive symptoms and no history of doctor-diagnosed depression at baseline. The fully adjusted Model $\mathrm{C}$ adjusts for age, sex, wave at follow-up, partnership, paid work, education, wealth, health behaviours, health conditions, functional limitations, waist circumference, season of blood collection at follow-up and an indicator of same seasonality across waves. Source: ELSA waves 6, 8, and 9. 
that hypovitaminosis D across waves was associated with elevated depressive symptoms were confirmed even when analyses were restricted to respondents with no elevated depressive symptoms and no history of doctordiagnosed depression at baseline (Model C).

\section{Discussion}

Cross-sectional associations between vitamin $\mathrm{D}$ insufficiency and depression or depressive symptoms are well documented but few studies have investigated this relationship longitudinally. To our knowledge, no prior studies have looked at the associations between changes in vitamin D levels and depressive symptoms. Using ELSA's longitudinal and large representative sample of older adults aged 50 years and older, we found that compared with participants with sustained sufficient levels of vitamin $\mathrm{D}$, those with persistent hypovitaminosis $\mathrm{D}$ over time have increased odds of reporting elevated depressive symptoms, even when a range of socio-economic and health characteristics, including baseline elevated depressive symptoms, are accounted for. However, our study does not provide evidence that 'becoming at risk' of hypovitaminosis D or 'being no longer at risk' is associated with changes in depressive symptoms.

Our results are in line with other observational longitudinal studies which found that baseline low vitamin $\mathrm{D}$ was associated with subsequent depression among older people ${ }^{25,28,29}$. Several direct and indirect biological mechanisms have been hypothesised to explain these associations, including the presence of Vitamin D receptors in areas of the brain associated to the development of depression, its role in the formation of serotonin, and its proposed anti-inflammatory effect ${ }^{31,32}$.

Our results also suggest that Vitamin D status is not stable over time for a considerable percentage of older people; however, in our study we found that changes in vitamin D levels were not associated with changes in elevated depressive symptoms. This finding warrants further studies on the effect of changes in Vitamin D levels on depression, and more specifically about the duration of such changes. In our study, where vitamin D level was assessed on two single time points, we cannot establish when and for how long respondents have become/are no longer at risk of hypovitaminosis D. Both factors, however, are likely to play an important role in understanding whether and how long it takes for changes in Vitamin D to affect depressive symptoms, as suggested by studies that have investigated the role of Vitamin D supplementation as prevention or treatment for later life depression. A randomised controlled trial conducted by Sanders and colleagues ${ }^{33}$, which investigated the effects of a yearly, large dose of 500,000 IU of vitamin D3 on depressive symptoms in women aged 70 years and older, concluded that although serum $25(\mathrm{OH}) \mathrm{D}$ levels were $41 \%$ higher in the women who received the vitamin D supplements versus the placebo after one year, no significant change in mood or mental well-being was found. Jorde and colleagues $^{34}$, however, found in their trial of overweight and obese men and women aged 21-70 years that there was evidence of modest improvement in depression scores after one year of vitamin D supplementation. More recent studies and systematic reviews ${ }^{35-39}$ continue to provide mixed findings to support the role of vitamin D supplementation in depression, depending on the target population, the duration, the definition of depression, as well as the dosage used.

Nonetheless, given seasonal changes in the levels of vitamin $\mathrm{D}$, supplementation may be important throughout the year, and in the winter in particular. Given the association of hypovitaminosis D with a number of indicators of poor health and musculoskeletal disorders, particularly among older adults, it is important to maintain sufficient levels of vitamin D over time, and this may be challenging when sun exposure is limited or inadequate. Small randomised trials also suggest that supplementation during winter with vitamin D might help seasonal mood disorders and improve well-being ${ }^{40-42}$.

The strengths of the study include the use of longitudinal data from a large and representative sample of community-dwelling men and women aged 50 years and older in England. We were also able to adjust our analyses for a wide range of socio-economic and health variables. Moreover, blood collection and analyses followed standardised protocols that ensure data quality. Our study, however, has some limitations and whilst this study is the first to assess changes in Vitamin D levels, we only had individual data pertaining to two timepoints. Therefore, we cannot ascertain the duration of hypovitaminosis D; this could be particularly important for those who 'became at risk' or 'were no longer at risk' of low vitamin D levels. As ELSA plans to continue to collect blood samples, future analyses will allow for a more nuanced and greater understanding of the dynamics of changes in Vitamin D and depressive symptoms. Moreover, the study may suffer from potential selection bias through eligibility criteria for the nurse visit, which was limited to those consenting to have their blood taken, and those who have remained in the study. However, if healthier respondents were more likely to both give blood and stay in the study, it is possible that our estimates may be conservative. Finally, ELSA does not collect information on the general use of vitamin D supplementation.

In conclusion, our study suggests a role of vitamin $\mathrm{D}$ in depression, with those with consistent hypovitaminosis D more likely to be depressed than those with high levels of vitamin D. Given the high prevalence of hypovitaminosis D and depression in later life, our findings might have significant public health implications. However, additional data from randomised controlled trials of Vitamin D supplementation are needed to ascertain if this could be a cost-effective strategy to prevent and reduce depression in later life.

\section{Methods}

Study population. We used data from the English Longitudinal Study of Ageing (ELSA), a multidisciplinary longitudinal survey representative of men and women aged 50 and over living in private households in England $^{43}$. The first interview took place in 2002/03, with biennial follow-up interviews and objective health examinations for all members (i.e. nurse visits) every 4 years until wave 6 . Across wave 8 and 9 , however, a new approach was taken when designing the nurse visit and two mutually exclusive subsets of members were selected: one was offered a nurse visit at wave 8 , and the other in wave 9. Full details of the study, its sampling 
design, and technical reports can be found elsewhere (http://www.elsa-project.ac.uk/). Analyses for this study used data from waves 6,8 , and 9 collected in 2012/13,2016/17, and 2018/19, respectively. Wave 6 of ELSA was the first to measure Vitamin D. In our analytical sample, we included participants who had a valid assessment of $25 \mathrm{OHD}$ at both baseline (wave 6) and follow-up (either wave 8 or 9) and measures of depression and the other covariates at both time points (please, see sections below). The analytical sample for the longitudinal analysis consisted of 3365 individuals. All participants gave written informed consent. The National Research Ethics Service (London Multicentre Research Ethics Committee [MREC/01/2/91]) has approved the ELSA study.

Assessment of 250HD. At Wave 6, of the 7730 who had a nurse visit, blood samples were obtained from 6206 participants. Blood samples were not taken from respondents who had a clotting or bleeding disorder (e.g., haemophilia or low platelets), had ever had a fit, were on anticoagulant drugs (e.g., warfarin therapy) or did not give their consent in writing. $25(\mathrm{OH}) \mathrm{D}$ concentrations were ascertained in 5870 . Almost $60 \%$ of these respondents $(\mathrm{N}=3472)$ also had a valid assessment of the vitamin $\mathrm{D}$ level at follow-up (either at Wave 8 or Wave 9). All blood samples were analysed at the Victoria Infirmary (Newcastle-upon-Tyne, United Kingdom). The vitamin $\mathrm{D}$ level was assessed by measuring the plasma level of $25(\mathrm{OH}) \mathrm{D}$. This has a circulating half-life of 2-3 weeks and is not influenced by changes in calcium and parathyroid hormone levels and is a reliable indicator of vitamin $\mathrm{D}$ status $^{44}$. Serum 25(OH)D levels were measured by the Diasorin Liaison immunoassay that detects both $25(\mathrm{OH})$ $\mathrm{D} 2$ and $25(\mathrm{OH}) \mathrm{D} 3$ and therefore, provides the total circulating $25(\mathrm{OH}) \mathrm{D}$ level. The assay for $25(\mathrm{OH}) \mathrm{D}$ has an analytical sensitivity (lower detection limit) of $7.5 \mathrm{nmol} / \mathrm{L}$. The detection limit represents the lowest measurable analyte level that can be distinguished from zero. All assays were performed in duplicate. The coefficient of variation ranged from 8.7 to $9.4 \%$. The laboratory performing the $25(\mathrm{OH}) \mathrm{D}$ analyses took part in the Internal and the Vitamin D External Quality Assessment Schemes (DEQAS). Based on the recommendations made by advisory bodies such as the European Food Safety Authority ${ }^{45}$ or the Institute of Medicine ${ }^{46}$ we categorised $25(\mathrm{OH}) \mathrm{D}$ levels using the truncated categorisation of $\leq 50 \mathrm{nmol} / \mathrm{L}$ (insufficient levels) and $>50 \mathrm{nmol} / \mathrm{L}$. In order to examine changes in Vitamin D levels between waves of the survey we distinguished between those with sufficient levels at both waves; those who became at risk (with sufficient levels at baseline but not at follow-up); those no longer at risk (with insufficient levels at baseline but with $25(\mathrm{OH}) \mathrm{D}$ levels $>50 \mathrm{nmol} / \mathrm{L}$ at follow-up) and those with insufficient levels of Vitamin D at both time points.

Assessment of depressive symptoms. Depressive symptoms were measured by a short eight-item version of the validated Centre for Epidemiologic Studies Depression (CES-D) Scale. The CES-D scale is not a diagnostic instrument for clinical depression but can be used to identify people "at risk" of depression in population-based studies ${ }^{47}$. This short version had good internal consistency at each wave (Cronbach's $\left.\alpha>0.95\right)$ and comparable psychometric properties to the full 20 -item CES- $\mathrm{D}^{48}$. Respondents were asked whether they had experienced any depressive symptoms, such as restless sleep or being unhappy in the week prior to interview. Respondents who reported four or more depressive symptoms in the week prior to the interview were classified as reporting elevated depressive symptoms ${ }^{49,50}$.

Covariates. A wide range of potential health, demographic, and socioeconomic confounders known to be associated with either depression or the likelihood of having low $25(\mathrm{OH}) \mathrm{D}$ levels were included. We controlled for baseline respondent's age, gender, partnership status, employment status, education, and quintiles of total net non-housing wealth. Age squared was not included in the model as adding this as a predictor variable did not result in a statistically significant improvement in model fit (p value for the likelihood ratio test was 0.341). Health behaviour measures included smoking status (non-smoker, former smoker, or current smoker) and selfreported physical activity (sedentary or low, moderate, or vigorous). Health measures included a summary score adding up the number of self-reported doctor diagnosed chronic conditions (high blood pressure, angina, heart attack, heart failure, heart murmur, hearth rhythm, diabetes, cancer, stroke, arthritis, lung diseases, or Parkinson's) as well as the sum of any difficulties with 13 basic (ADL) and instrumental (IADL) activities of daily living. A history of doctor diagnosed depression was established by asking participants whether they were ever told by a doctor they had depression. Waist circumference was categorised into three main groups using sex-specific thresholds: low ( $<94 \mathrm{~cm}$ for men; $<80 \mathrm{~cm}$ for women), medium ( $\geq 94 \mathrm{~cm}$ and $<102 \mathrm{~cm}$ for men; $\geq 80 \mathrm{~cm}$ and $<88$ for women), and high ( $\geq 102 \mathrm{~cm}$ for men; $\geq 88 \mathrm{~cm}$ for women). We also included the season when the follow-up sample was collected, and an indicator capturing whether follow-up blood sample was collected in the same grouped season (summer and autumn or winter and spring) or not. Finally, we controlled for an indicator of the wave of the follow-up interview.

Statistical analysis. Following descriptive findings to explore the baseline characteristics of the study population, longitudinal logistic regression models were used to determine whether changes in vitamin D levels were associated with depression. Following the unadjusted model (Model 1), a further six models adjusted for potential confounders were performed, with each subsequent model including variables from the previous model. Model 2 adjusted for age, sex, and the wave at follow-up. Model 3 further adjusted for partnership, employment status, education, and wealth; Model 4, for health behaviours, health conditions, and functional limitations; Model 5, for waist circumference; Model 6, for elevated baseline depressive symptoms and history of doctor-diagnosed depression; and finally, Model 7, for the season of blood collection on follow-up, and the seasonal indicator variable. All analyses were carried out using Stata SE version 15.1.

Sensitivity analyses. Sensitivity analyses were conducted to test the robustness of our models. In particular, we repeated our analyses (a) taking into account changes in respondents' partnership, wealth, and health 
instead of considering these variables only at baseline; (b) restricting the sample to those who had had their blood sample taken in the same seasons at both baseline and follow-up; and (c) restricting the sample to those without a history of doctor-diagnosed depression or elevated depressive symptoms at baseline to rule out potential reverse causation of the associations. We also repeated analyses by using as the main variable of interest the number of times 'at risk' of having insufficient levels of Vitamin D (distinguishing between never, once, and at both waves) as well as considering baseline hypovitaminosis $\mathrm{D}$ and absolute changes in Vitamin $\mathrm{D}$ levels over time.

\section{Data availability}

The data were made available through the UK Data Archive.

Received: 17 December 2020; Accepted: 30 March 2021

Published online: 08 April 2021

\section{References}

1. World Health Organization. Depression and Other Common Mental Disorders: Global Health Estimates (World Health Organization, 2017).

2. Stewart, W. F. et al. Cost of lost productive work time among US workers with depression. JAMA 289(23), 3135-3144 (2003).

3. Vasiliadis, H.-M. et al. The excess healthcare costs associated with depression and anxiety in elderly living in the community. Am. J. Geriatr. Psychiatry 21(6), 536-548 (2013).

4. Kessler, R. C. The costs of depression. Psychiatr. Clin. N. Am. 35(1), 1-14 (2012).

5. Stumpf, W. et al. Brain target sites for 1,25-dihydroxyvitamin D3. Science 215(4538), 1403-1405 (1982).

6. Milaneschi, Y. et al. The association between low vitamin D and depressive disorders. Mol. Psychiatry 19(4), 444-451 (2014).

7. Nair, R. \& Maseeh, A. Vitamin D: The "sunshine" vitamin. J. Pharmacol. Pharmacother. 3(2), 118-126 (2012).

8. Prentice, A. Vitamin D deficiency: a global perspective. Nutr. Rev. 66(suppl_2), S153-S164 (2008).

9. Boucher, B. J. The problems of vitamin d insufficiency in older people. Aging Dis. 3(4), 313-329 (2012).

10. Mithal, A. et al. Global vitamin D status and determinants of hypovitaminosis D. Osteoporos. Int. 20(11), 1807-1820 (2009).

11. MacLaughlin, J. \& Holick, M. F. Aging decreases the capacity of human skin to produce vitamin D3. J. Clin. Investig. 76(4), 1536-1538 (1985).

12. Holick, M. F. Vitamin D: Extraskeletal health. Rheum Dis Clin N. Am 38(1), 141-160 (2012).

13. Sahota, O. Understanding vitamin D deficiency. Age Ageing 43(5), 589-591 (2014).

14. Reid, I. R. \& Bolland, M. J. Skeletal and nonskeletal effects of vitamin D: Is vitamin D a tonic for bone and other tissues?. Osteoporos. Int. 25(10), 2347-2357 (2014).

15. Hoogendijk, W. J. et al. Depression is associated with decreased 25-hydroxyvitamin D and increased parathyroid hormone levels in older adults. Arch. Gen. Psychiatry 65(5), 508-512 (2008).

16. Zhao, G. et al. No associations between serum concentrations of 25 -hydroxyvitamin D and parathyroid hormone and depression among US adults. Br. J. Nutr. 104(11), 1696-1702 (2010).

17. Pan, A. et al. Association between depressive symptoms and 25 -hydroxyvitamin $\mathrm{D}$ in middle-aged and elderly Chinese. J. Affect. Disord. 118(1), 240-243 (2009).

18. Stewart, R. \& Hirani, V. Relationship between vitamin D levels and depressive symptoms in older residents from a national survey population. Psychosom. Med. 72(7), 608-612 (2010).

19. de Oliveira, C., Hirani, V. \& Biddulph, J. P. Associations between vitamin D levels and depressive symptoms in later life: Evidence From the English Longitudinal Study of Ageing (ELSA). J Gerontol. Ser. A 73(10), 1377-1382 (2017).

20. Hoang, M. T. et al. Association between low serum 25 -hydroxyvitamin D and depression in a large sample of healthy adults: The Cooper Center Longitudinal Study. Mayo Clin. Proc. 86(11), 1050-1055 (2011).

21. Ganji, V. et al. Serum vitamin D concentrations are related to depression in young adult US population: The Third National Health and Nutrition Examination Survey. Int. Arch. Med. 3, 29 (2010).

22. Lee, D. M. et al. Lower vitamin D levels are associated with depression among community-dwelling European men. J. Psychopharmacol. 25(10), 1320-1328 (2011).

23. Lapid, M. I., Cha, S. S. \& Takahashi, P. Y. Vitamin D and depression in geriatric primary care patients. Clin. Interv. Aging 8, 509-514 (2013).

24. Han, B. et al. Low serum levels of vitamin D are associated with post-stroke depression. Eur. J. Neurol. 22(9), 1269-1274 (2015).

25. Anglin, R. E. S. et al. Vitamin D deficiency and depression in adults: Systematic review and meta-analysis. Br. J. Psychiatry 202(2), 100-107 (2013).

26. Toffanello, E. D. et al. Serum 25-hydroxyvitamin d and the onset of late-life depressive mood in older men and women: The ProVA study. J. Gerontol. Ser. A Biol. Sci. Med. Sci. 69(12), 1554-1561 (2014).

27. Tolppanen, A.-M. et al. The association of serum 25-hydroxyvitamin D3 and D2 with depressive symptoms in childhood-A prospective cohort study. J. Child Psychol. Psychiatry 53(7), 757-766 (2012).

28. Williams, J. A. et al. Low 25 -hydroxyvitamin D concentrations predict incident depression in well-functioning older adults: The health, aging, and body composition study. J. Gerontol. Ser. A 70(6), 757-763 (2014).

29. Ronaldson, A. et al. Prospective associations between vitamin D and depression in middle-aged adults: Findings from the UK Biobank cohort. Psychol. Med. https://doi.org/10.1017/S0033291720003657 (2020).

30. Andersen, R. et al. Seasonal changes in vitamin D status among Danish adolescent girls and elderly women: The influence of sun exposure and vitamin D intake. Eur. J. Clin. Nutr. 67(3), 270-274 (2013).

31. Bertone-Johnson, E. R. Vitamin D and the occurrence of depression: Causal association or circumstantial evidence?. Nutr. Rev. 67(8), 481-492 (2009).

32. de Oliveira, C. et al. Vitamin D and inflammatory markers: Cross-sectional analyses using data from the English Longitudinal Study of Ageing (ELSA). J. Nutr. Sci. 6, el (2017).

33. Sanders, K. M. et al. Annual high-dose vitamin D3 and mental well-being: Randomised controlled trial. Br. J. Psychiatry 198(5), 357-364 (2011).

34. Jorde, R. et al. Effects of vitamin D supplementation on symptoms of depression in overweight and obese subjects: Randomized double blind trial. J. Intern. Med. 264(6), 599-609 (2008).

35. Alavi, N. M. et al. Effect of vitamin D supplementation on depression in elderly patients: A randomized clinical trial. Clin. Nutr. 38(5), 2065-2070 (2019).

36. de Koning, E. J. et al. Vitamin D supplementation for the prevention of depression and poor physical function in older persons: The D-Vitaal study, a randomized clinical trial. Am. J. Clin. Nutr. 110(5), 1119-1130 (2019).

37. Kjærgaard, M. et al. Effect of vitamin D supplement on depression scores in people with low levels of serum 25-hydroxyvitamin D: Nested case-control study and randomised clinical trial. Br. J. Psychiatry 201(5), 360-368 (2012). 
38. Vellekkatt, F. \& Menon, V. Efficacy of vitamin D supplementation in major depression: A meta-analysis of randomized controlled trials. J. Postgrad. Med. 65(2), 74-80 (2019).

39. Shaffer, J. A. et al. Vitamin D supplementation for depressive symptoms: A systematic review and meta-analysis of randomized controlled trials. Psychosom. Med. 76(3), 190-196 (2014).

40. Lansdowne, A. T. \& Provost, S. C. Vitamin D3 enhances mood in healthy subjects during winter. Psychopharmacology 135(4), 319-323 (1998).

41. Gloth, F. M. III., Alam, W. \& Hollis, B. Vitamin D vs broad spectrum phototherapy in the treatment of seasonal affective disorder. J. Nutr. Health Aging 3(1), 5-7 (1999).

42. Vieth, R. et al. Randomized comparison of the effects of the vitamin D3 adequate intake versus $100 \mathrm{mcg}$ (4000 IU) per day on biochemical responses and the wellbeing of patients. Nutr. J. 3, 8 (2004).

43. Zaninotto, P. \& Steptoe, A. English longitudinal study of ageing. In Encyclopedia of Gerontology and Population Aging (eds Gu, D. \& Dupre, M. E.) 1-7 (Springer, 2019).

44. Holick, M. F. Vitamin D status: Measurement, interpretation, and clinical application. Ann. Epidemiol. 19(2), 73-78 (2009).

45. EFSA Panel on Dietetic Products, N.A. Dietary reference values for vitamin D. EFSA J. 14(10), e04547 (2016).

46. Ross, A. C. et al. The 2011 report on dietary reference intakes for calcium and vitamin D from the institute of medicine: What clinicians need to know. J. Clin. Endocrinol. Metab. 96(1), 53-58 (2011).

47. Radloff, L. S. The CES-D Scale: A self-report depression scale for research in the general population. Appl. Psychol. Meas. 1(3), 385-401 (1977).

48. Karim, J. et al. Validation of the eight-item Center for Epidemiologic Studies Depression Scale (CES-D) among older adults. Curr. Psychol. 34(4), 681-692 (2015).

49. Turvey, C. L., Wallace, R. B. \& Herzog, R. A revised CES-D measure of depressive symptoms and a DSM-based measure of major depressive episodes in the elderly. Int. Psychogeriatr. 11(2), 139-148 (1999).

50. Zivin, K. et al. Depression among older adults in the United States and England. Am. J. Geriatri. Psychiatry 18(11), 1036-1044 (2010).

\section{Acknowledgements}

The data were made available through the UK Data Archive. The English Longitudinal Study of Ageing was developed by a team of researchers based at University College London, NatCen Social Research, the Institute for Fiscal Studies, the University of Manchester and the University of East Anglia. The data were collected by NatCen Social Research. The funding is currently provided by the National Institute of Aging in the United States, and a consortium of UK government departments co-ordinated by the National Institute for Health Research. Funding has also been received by the Economic and Social Research Council.

\section{Author contributions}

G.D.G., C.d.O., and J.B. made substantial contributions to the conception and design of the study. G.D.G. analysed the data. P.Z. and J.B. contributed to the analysis and interpretation of data. G.D.G. drafted the manuscript. All authors have revised the article and have approved the final version of the manuscript.

\section{Funding}

This research did not receive any funding from agencies in the public, commercial, or not-for-profit sectors.

\section{Competing interests}

The authors declare no competing interests.

\section{Additional information}

Correspondence and requests for materials should be addressed to G.D.G.

Reprints and permissions information is available at www.nature.com/reprints.

Publisher's note Springer Nature remains neutral with regard to jurisdictional claims in published maps and institutional affiliations.

(c) (i) Open Access This article is licensed under a Creative Commons Attribution 4.0 International cc) License, which permits use, sharing, adaptation, distribution and reproduction in any medium or format, as long as you give appropriate credit to the original author(s) and the source, provide a link to the Creative Commons licence, and indicate if changes were made. The images or other third party material in this article are included in the article's Creative Commons licence, unless indicated otherwise in a credit line to the material. If material is not included in the article's Creative Commons licence and your intended use is not permitted by statutory regulation or exceeds the permitted use, you will need to obtain permission directly from the copyright holder. To view a copy of this licence, visit http://creativecommons.org/licenses/by/4.0/.

(c) The Author(s) 2021 